\title{
A Phase Aberration Correction Method for Ultrasound Imaging
}

\author{
Mustafa Karaman, Student Member, IEEE, Abdullah Atalar, Senior Member, IEEE \\ Hayrettin Köymen, Senior Member, IEEE, and Matthew O'Dōnnell, Fellow, IEEE
}

\begin{abstract}
A computationally efficient method for phase aberration correction in ultrasound imaging is presented. The method is based on time delay estimation via minimization of the sum of absolute differences between radio frequency samples of adjacent array elements. Effects of averaging estimated aberration patterns over scan angles, and truncation to a single bit wordlength are examined. Phase distortions due to near-field inhomogeneities are simulated using silicone rubber aberrators. Performance of the method is tested using experimental data. Simulation studies addressing different factors affecting efficiency of the method, such as the number of iterations, window length, and the number of scan angles used for averaging, are presented. Images of a standard resolution phantom are reconstructed and used for qualitative testing.
\end{abstract}

\section{INTRODUCTION}

$\mathbf{P}$ HASED array ultrasound sector images are reconstructed by employing beamforming operations both in transmit and receive modes. Timing considerations for beamforming are usually based on the assumption of a constant velocity. However, this assumption is not valid in general since the sound velocity varies in different tissues. Changes in sound velocity, especially in the near field, cause significant phase errors in beamforming. These phase errors result in increased sidelobes, degradation in resolution, and geometrical distortion [1].

To insure optimal imaging, phase errors must be estimated and corrected adaptively in real-time. Use of a beacon signal for phase estimation, a conventional method in many coherent imaging systems, may not be possible in noninvasive medical imaging applications. A practical method must obtain accurate estimates of phase errors using the signals from line targets and/or diffuse scatterers.

Phase aberration correction methods have been reported for a nonuniform aberrating layer with a deterministic target [2], for a uniform aberrating layer with a priori information about the layer thickness [3], and for nonuniform aberrating structures with random reflector distributions [4]-[7]. Recently, two-dimensional studies have been reported [8]-[10]. Also, there exist time delay estimation techniques [11] which

Manuscript received October 7, 1992; revised February 22, 1993; accepted February 26, 1993.

M. Karaman, A. Atalar, and H. Köymen are with the Department of Electrical and Electronics Engineering, Bilkent University, Ankara 06533, Turkey.

M. O'Donnell is with the Department of Electrical Engineering and Computer Science, University of Michigan, Ann Arbor, MI 48109-2122.

IEEE Log Number 9209516. involve extensive computations, and hence, are more suitable for off-line phase correction. For real-time ultrasound imaging, however, the computational efficiency of the correction algorithm is crucial.

In this paper, we present an alternative phase aberration correction technique with low computational complexity. The relative phase errors of adjacent array channels are estimated effectively through minimization of the sum of absolute differences (SAD) between two radio frequency (RF) samples. In order to increase the accuracy and convergence performance of the SAD technique, we employ weighted averaging of estimated patterns over a number of scan angles. The weighting coefficient for each angle is chosen as the average magnitude of the echo signal received at this angle. To further increase the computational efficiency of the technique, the wordlength is truncated to $1 \mathrm{~b}$.

In the next section, the phase aberration correction method based on SAD and its derivatives are described. Performance analysis of the proposed method is presented in Section III. Efficiency of the aberration correction schemes are discussed in Section IV by means of additional simulation studies. The results and conclusions are outlined in Section V.

\section{METHOD}

The SAD is used as a cost function in minimization problems because of its low computational complexity [12]-[14]. We employ the SAD for estimation of the relative phase difference between two sampled signals. The SAD of RF echo samples received from adjacent array channels, over a time window of length $M$, is expressed as a function of shift index, $r_{n}^{i}$, as

$$
\epsilon\left(r_{n}^{i}\right)=\sum_{k=1}^{M}\left|s_{n}^{i}(k)-s_{n-1}^{i}\left(k+r_{n}^{i}\right)\right|
$$

where $s_{n}^{i}(k)$ is the $k$ th sample of the $n$th element's signal at the $i$ th scan angle. Typically, $s_{n}^{i}(\cdot)$ and $s_{n-1}^{i}(\cdot)$ are approximately shifted versions of each other. The value of $r_{n}^{i}$, which minimizes this expression, is found for every $n$ and $i$. The relative aberration delay for the $n$th element at the $i$ th scan angle, $\alpha_{n}^{i}$, is given by

$$
\alpha_{n}^{i}=T_{s} r_{n}^{i}-\gamma_{n}^{i}
$$

where $T_{s}$ is the sampling period, and $\gamma_{n}^{i}$ is the relative focusing delay. The aberration delay time, $\tau_{n}^{i}$ for the $n$th 
element at the $i$ th scan angle is the cumulative summation of the relative aberration delays:

$$
\tau_{n}^{i}=\sum_{j=1}^{n} \alpha_{j}^{i}
$$

Time delay estimation using simple SAD minimization can be used to measure phase distortions due to nonuniform aberrating layers. However, scatterers in the direction of sidelobes may also contribute to phase errors, which are referred to as phase aberration due to the "multipath and scattering" [15]. Depending on the scatterer distribution over the isoplanatic patch where the phase estimation is carried out, the effect of multipath and scattering may not cancel out, resulting in focusing errors. Such phase errors may affect the accuracy and convergence performance of the aberration estimation. In order to reduce these errors, the estimation can be performed over a lateral window as well as over a time (axial) window. This can be achieved by minimizing of the SAD over a two-dimensional window. Another possibility is to minimize the SAD and average the weighted estimates over a number of scan angles. Computer simulations show that the performance of these schemes is equivalent [16], while the latter requires a relatively small number of arithmetic operations.

Because the echo signal is not uniform over angles, averaging of the estimated aberration delays over a two-dimensional window needs proper weighting. The weight coefficient of the $n$th array element, $w_{n}^{i}$, for the $i$ th scan angle is defined as the average magnitude of the echo signal received from this element, i.e.:

$$
w_{n}^{i}=\frac{1}{M} \sum_{k=1}^{M}\left|s_{n}^{i}(k)\right| .
$$

Either a unique weighting for each element or a single weighting bound on the average magnitude over all elements can be used. This weighting scheme biases the estimation to scan angles where relatively strong reflections are detected. Also, it requires a simple computation based on addition. The weighted average of the estimated aberration delays is expressed as

$$
\bar{\tau}_{n}=\sum_{i=1}^{K} w_{n}^{i} \tau_{n}^{i} / \sum_{i=1}^{K} w_{n}^{i}
$$

where $K$ is the number of scan angles used for averaging, and $\bar{\tau}_{n}$ is the averaged aberration delay for the $n$th element.

Computational efficiency of the SAD technique can be further increased using a shorter wordlength. The phase information of RF signals can be adequately represented by zero crossings of the carrier. The relative phase difference between two RF signals, then, can be easily detected from shifts in their zero crossings. A single bit wordlength is sufficient for representation of the zero crossings of the sampled signal. Hence, the SAD technique can be used on RF samples properly quantized to a single bit, where each absolute difference operation reduces to a bitwise EXCLUSIVE-OR $(\oplus)$. In this case, the $\mathrm{SAD}, \epsilon^{\prime}\left(r_{n}^{i}\right)$, is given by

$$
\epsilon^{\prime}\left(r_{n}^{i}\right)=\sum_{k=1}^{M}\left[\tilde{s}_{n}^{i}(k)\right] \oplus\left[\tilde{s}_{n-1}^{i}\left(k+r_{n}^{i}\right)\right]
$$

where $\tilde{s}_{n}^{i}(k)$ and $\tilde{s}_{n-1}^{i}(k)$ are the one bit representations of echo samples $s_{n}^{i}(k)$ and $s_{n-1}^{i}(k)$, respectively. Reduction of the wordlength to a single bit can cause poor convergence in phase estimation from speckle noise. This effect can be avoided, however, by using averaging. No weights are used to further simplify computations.

\section{Performance Analysis}

To test the performance of different phase aberration correction techniques, computer simulations are performed using actual RF data sets acquired from a standard graphite-gel AIUM resolution phantom with and without aberrators present. This phantom simulates tissue while providing thin wire reflectors emulating a beacon. All data were acquired with a conventional 64-element phased array transducer operating at $3.3 \mathrm{MHz}$ [4], [17]. The RF A-scans were recorded for every possible combination of transmitter and receiver elements. Each A-scan was digitized after appropriate time gain compensation at a sampling rate of $17.76 \mathrm{MHz}$ with $10-\mathrm{b}$ analog-to-digital conversion. Three data sets were obtained using three plates, made of RTV silicone rubber, placed between the transducer array and the phantom. The first plate produces only a very small defocusing, and hence, is used as the control plate. This defocusing effect is minor and can be neglected [17]. The second plate causes phase distortions less than $\pi$, whereas the phase distortion imposed by the third plate varies within $2 \pi$. In the rest of this paper, we refer to these plates as Plate 0 , Plate 1 , and Plate 2 , respectively. Image reconstruction and performance evaluation studies are carried out by digitally processing the recorded data to emulate the operation of a real-time digital imaging system.

Performance of the SAD method is quantitatively tested and compared with the cross correlation technique [4]. For this purpose, the rms error between the estimated and the actual aberration patterns are obtained for different parameters affecting the performance of the correction method, such as the number of iterations, the window length used for SAD computation, and the number of scan angles used for averaging. RMS error computations are carried out as follows:

$$
\text { RMS Error }=\sqrt{\frac{1}{N} \sum_{n=1}^{N}\left(\tau_{n}-\tau_{n}^{a}\right)^{2}}
$$

where $N$ is the number of array elements, and, $\tau_{n}$ and $\tau_{n}^{a}$ denote the estimated and the actual aberration delays for $n$th element, respectively.

Phase aberration patterns estimated from the beacon signals using full word cross correlation represent the gold standard and are assumed to be the actual patterns [17]. In measurement of the actual patterns, the transmit focus and the estimation window are fixed at $80 \mathrm{~mm}$ which corresponds to the location of the beacon. The original rms phase errors of Plate 0, Plate 1, 


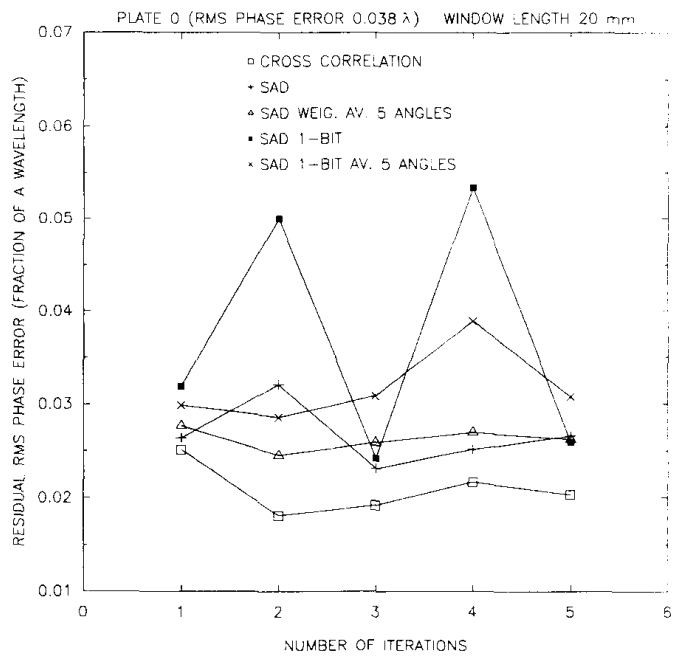

(a)

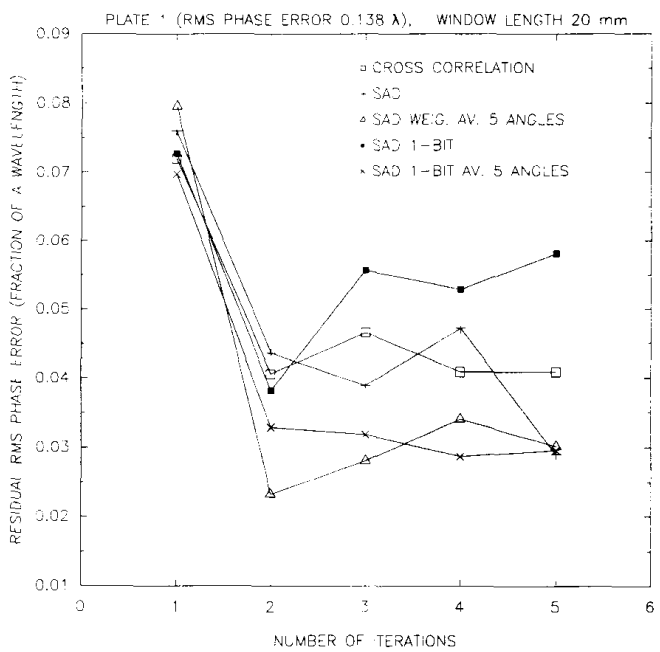

(b)

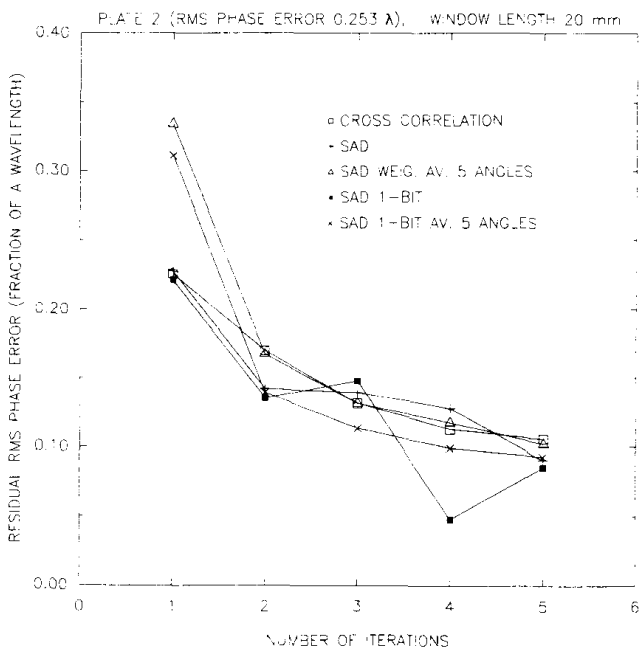

(c)

Fig. 1. Convergence and accuracy performance of phase correction schemes for different aberrators. (a) Plate 0. (b) Plate 1. (c) Plate 2.

and Plate 2 are $0.038,0.138$, and $0.253 \lambda$, respectively, where $\lambda$ is the wavelength of the $3.33-\mathrm{MHz}$ ultrasound signal.

All phase aberration estimations other than the gold standard are based on RF signals from diffuse scatterers. The aberration profile is estimated from scan angles nearly normal to the array face. The transmit focal length is fixed to $80 \mathrm{~mm}$, and the window used for cross correlation and SAD is located $60 \mathrm{~mm}$ away from the array. This region of the phantom consists of diffuse scatterers only. The sampling rate of each RF data set is increased by 8 times using bandpass interpolation. Hence, phase aberration correction and beamforming operations are performed with about a 142-MHz sampling rate, which corresponds to a phase accuracy of about $2 \pi / 43$.

To compare techniques at different aberration levels, and to investigate their convergence characteristics, the residual rms error after correction versus the number of iterations was obtained for each data set (see Fig. 1). Each iteration cycle consists of transmit beamforming with corrected delays, and aberration estimation. Since all RF A-scans were recorded for every possible combination of transmitter and receiver elements, transmit beamforming with updated phase correction is emulated at every iteration step. Transmit beamforming is achieved by summing the contributions of all transmit elements. The RF signal at each receive element is the sum of 64 transmitter signals delayed with corresponding transmit focusing delays. The aberration estimation is carried out on these received RF signals. At each iteration step, transmit focusing delays are updated using the aberration delays estimated in the previous iteration. In the first iteration cycle, the aberration delay of each element is assumed to be zero.

In Fig. 1, five different estimation schemes are presented. The first one uses normalized cross correlation of RF samples 
between the adjacent channels. Computations of the normalized correlation used in our simulations are carried out as follows:

$$
\frac{\begin{array}{l}
C\left(r_{n}^{i}\right)= \\
\sum_{k=1}^{M}\left[s_{n}^{i}(k)-S_{n}^{i}\right]\left[s_{n-1}^{i}\left(k+r_{n}^{i}\right)-S_{n-1}^{i}\right]
\end{array}}{\sqrt{\sum_{k=1}^{M}\left[s_{n}^{i}(k)-S_{n}^{i}\right]^{2} \sum_{k=1}\left[s_{n-1}^{i}\left(k+r_{n}^{i}\right)-S_{n-1}^{i}\right]^{2}}}
$$

where $S_{n}^{i}$ and $S_{n-1}^{i}$ are the mean values of $s_{n}^{i}(\cdot)$ and $s_{n-1}^{i}(\cdot)$, respectively, computed over the corresponding window. The other parameters are the same as those used in the SAD expression given in (1). The other four correction schemes are applications of SAD with different parameters as indicated in the figures.

Fig. 1 shows that phase errors can be corrected iteratively. Three iteration cycles are sufficient for reasonably accurate estimation of phase errors. The residual rms errors, after three correction cycles, for aberration patterns associated with the Plate 0, Plate 1, and Plate 2 are about 2.5, 4, and $10 \%$ of a wavelength, respectively. These values correspond to about 1,2 , and 4 units of phase delay ( 1 unit $=2 \pi / 43$ ). The simple SAD minimization with full word has some convergence problems. The convergence performance of 1 b SAD is even worse. Averaging, however, eliminates the convergence problem. It should be noted that averaging causes greater phase errors in the first iteration. The full word SAD with weighted averaging shows a better performance compared to other correction schemes. Overall performance of the 1-b SAD scheme with averaging is comparable to that of the full word cross correlation and full word SAD.

Fig. 2 depicts the sensitivity of the phase correction schemes to window size. The figure indicates, as expected, that the residual $\mathrm{rms}$ phase error decreases as the window size increases. It is observed that averaged estimators perform better than cross correlation at small window sizes. Furthermore, the window size requirements of 1-b SAD estimators are comparable to that of full word estimators. For example, their accuracy is comparable for a $20 \mathrm{~mm}$ window.

Proper selection of the number of angles for averaging is critical. It must be large enough to obtain a stable estimate in a few iterations. On the other hand, it must be small enough to confine the region to the vicinity of particular reflecting structures and avoid the effects of other scatterers. Also, it must be small enough to maintain computational efficiency. To choose a proper value for the number of angles to average, the full word SAD with weighted averaging and 1-b SAD with simple averaging are tested for different numbers of scan angles (see Fig. 3). The angular separation of two scan lines is about $0.7 \mathrm{deg}$ which corresponds to 128 scan lines per 90 deg sector. This combination is typical in real-time ultrasonic scanners.

It is observed from Fig. 3 that the residual phase error decreases as the number of scan angles used in averaging increases from one to eight. The figure also depicts that significant improvements are not obtained for more than five

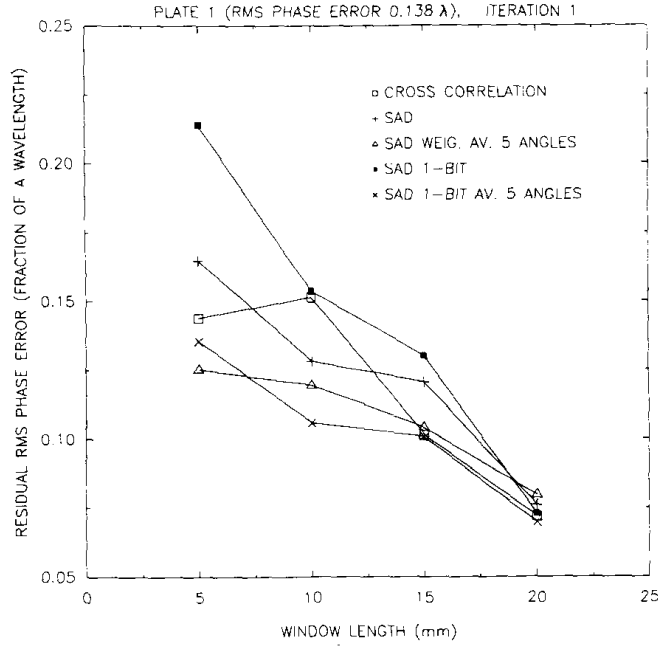

Fig. 2. Effect of window size on the accuracy of phase estimation techniques for Plate 1.

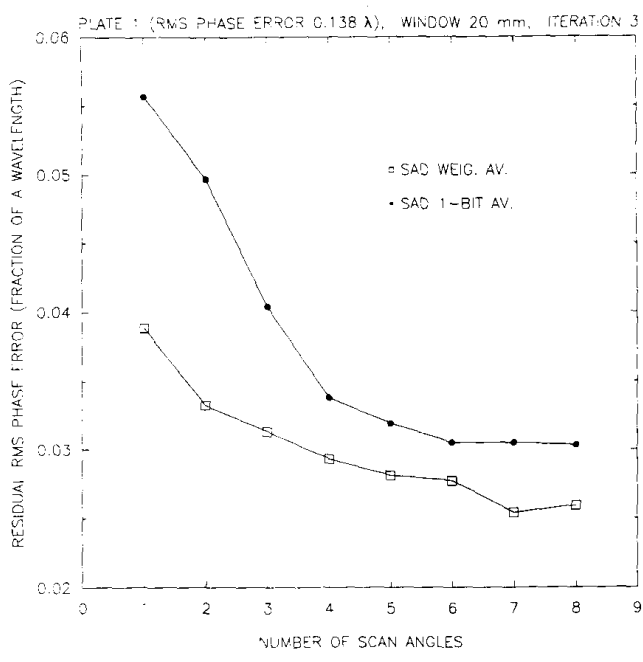

Fig. 3. Effect of the number of scan angles used for the averaging of phase estimates on the convergence and accuracy performances of full word SAD and the SAD with a single bit wordlength. The data set used in this figure is Plate 1.

scan angles. Therefore, for practical implementation reasons, SAD analysis can be restricted to only five scan angles without significant reduction in the quality of phase error estimation.

To test overall image quality, sector images of the phantom are reconstructed using both cross correlation and SAD correction schemes. Fig. 4 depicts the control, aberrated and the corrected images displayed on a purely logarithmic scale over a 50-dB dynamic range. A section of the sector image of the phantom is shown in the figure. This section includes a point reflector, a cyst-like structure, and speckle patterns, and hence, is a small sized image suitable for qualitative testing. Each image has 116 by 116 pixels corresponding to about a $26 \mathrm{~mm}$ by $26 \mathrm{~mm}$ spatial region. In reconstruction of the images, 128 

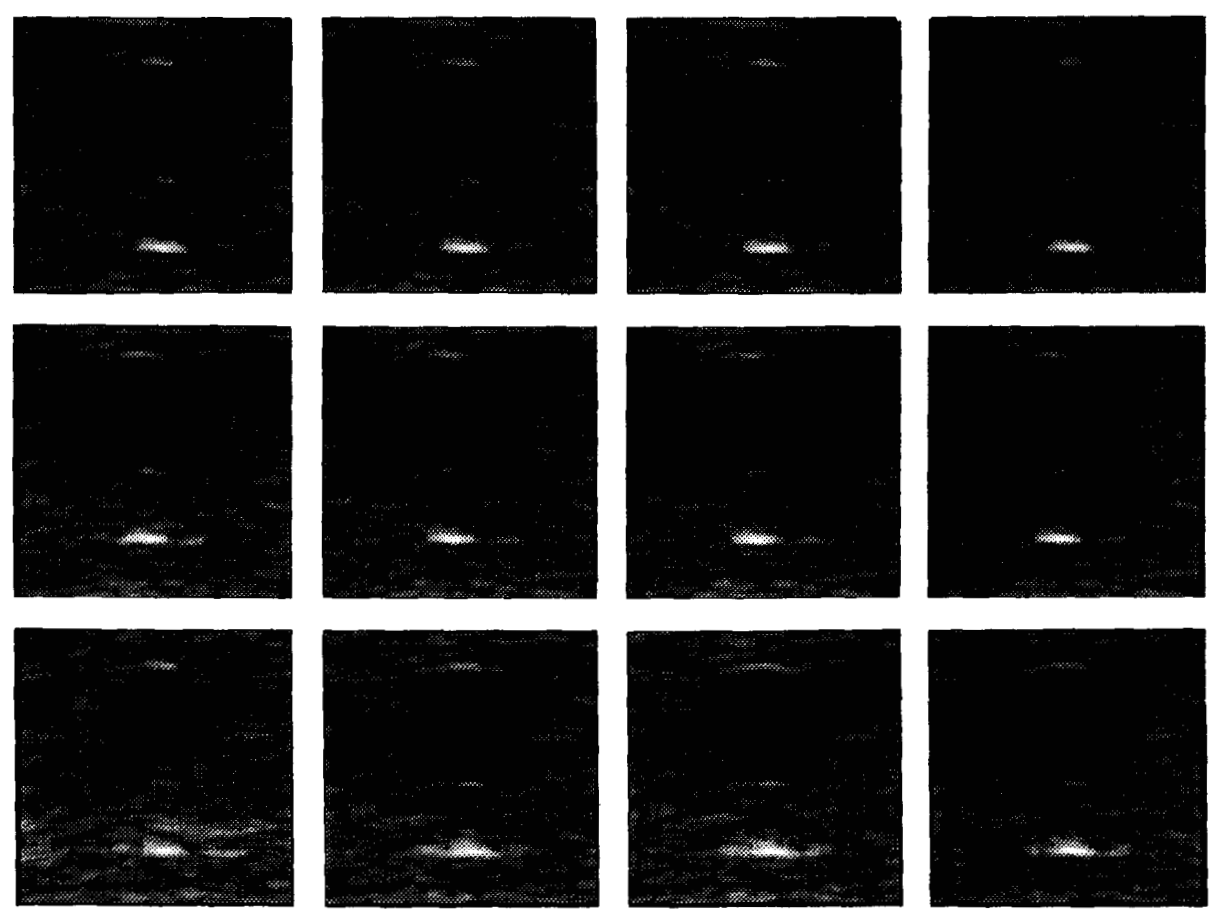

Fig. 4. Images of a section of the phantom. (a) Control and aberrated images. The corrected images using (b) full word cross correlation, (c) full word SAD with weighted averaging of estimates over five scan angles, and (d) SAD with 1-b wordlength and averaging of estimates over five scan angles. The images in the first, second, and third rows correspond to the data sets associated with Plate 0 , Plate 1 , and Plate 2 , respectively.

scan lines per 90-deg sector are used, transmit focus is fixed at $80 \mathrm{~mm}$ on each scan line, and dynamic receive focusing is employed. The point reflector seen in the images is located at a distance of about $80 \mathrm{~mm}$ away from the array.

For each corrected image, the transmit and receive focus delays are updated using the phase aberration pattern estimated in three iterations from diffuse scatterers over scan angles nearly normal to the aperture face. The window length used for cross correlation and SAD are $20 \mathrm{~mm}$. It is observed from the images shown in Fig. 4 that the performance of the full word $S A D$ with weighted averaging of estimates over five scan angles, compares favorably to that of the cross correlation method. In addition, image quality of the SAD with 1-b wordlength and averaging of estimates over five scan angles is as good as full word SAD and full word cross correlation.

Comparison of the control image with its corrected versions indicates that no artificial distortion is generated. However, it is observed that corrected versions of aberrated images are not the same as the control image. This is because the RTV plates also produce amplitude distortions across the array, in addition to simple phase aberrations [17]. Amplitude variations degrade beam quality, and hence, decrease the covariance of RF signals. The decrease in covariance limits the accuracy of phase estimation. Furthermore, aberration correction even with perfect phase compensation cannot produce an image which is an exact replica of the control image due to these amplitude distortions.

\section{Discussion}

The best performance of both SAD- and correlation-based aberration correction techniques can be obtained by estimating the phase from beacon signals or signals reflected by a point reflector, with the transmit focus placed at the center of the estimation window. The simulation studies presented in the previous section depicts the typical performance of methods since aberration estimation is performed on RF signals reflected by diffuse scatterers, and also, the transmit focus (at $80 \mathrm{~mm}$ ) and the estimation window location (at $60 \mathrm{~mm}$ ) do not coincide.

To illustrate the accuracy and convergence performance of aberration correction methods for different speckle data and different focusing schemes, the mean and standard deviation of residual $\mathrm{rms}$ phase error over ten trials are presented in Fig. 5. (a) and (b), respectively. The aberrated RF data set associated with Plate 1 is used to obtain these results. The mean and standard deviations are given in separate figures for the sake of clarity. Five of these trials are obtained on five different, nonadjacent scan lines with transmit focus at $80 \mathrm{~mm}$ and estimation window at $60 \mathrm{~mm}$, and the other five trials are obtained on the same scan lines of the previous five trials, with transmit focus at $60 \mathrm{~mm}$ and estimation window at 60 $\mathrm{mm}$. Therefore, the repeated ten trials involves five different speckle data and two different combinations of transmit focus and window location.

The simulation results shown Fig. 1. (b) and Fig. 5. (a) are in close agreement. Fig. 5. (b) shows that standard deviation of 


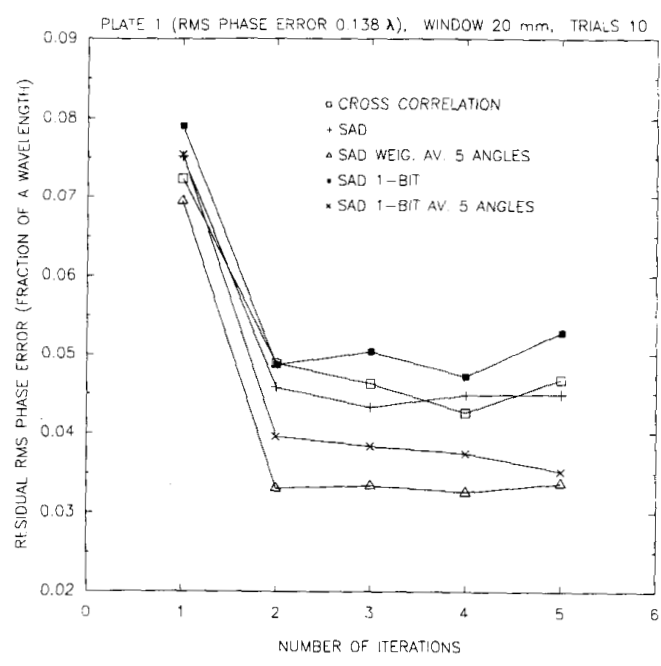

(a)

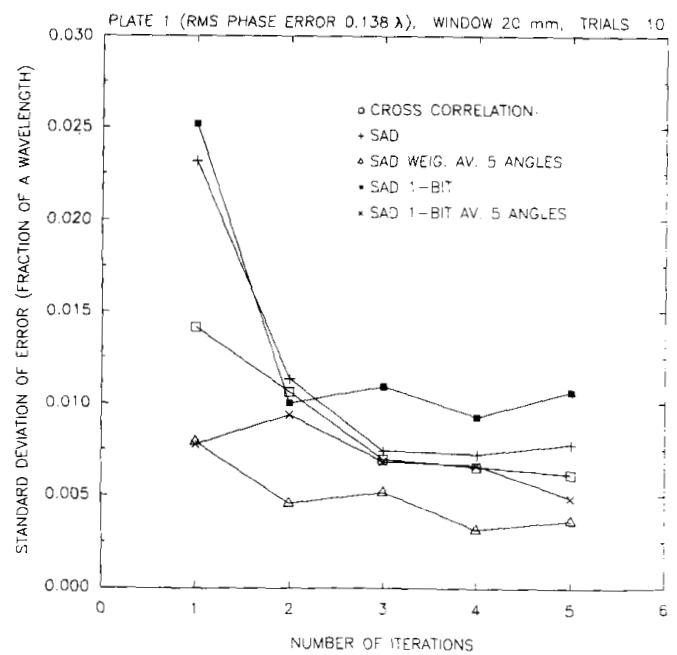

(b)

Fig. 5. Convergence and accuracy performance of phase correction schemes for Plate 1 using repeated trials on different speckle lines with different combinations of transmit focus and window location. (a) Mean and (b) standard deviation of the residual ms phase error of 10 trials.

the residual rms error for each estimation scheme is less than 1 $\%$ of a wavelength after two iterations. The standard deviations of the averaged SAD estimators are less than that of the others. The 1-b SAD estimator has the highest standard deviation, in accordance with the instability also observed in Fig. 1. These simulation results of repeated trials with different parameters further implies that the results of the previous section are more general than the specific experimental cases presented.

The performance of aberration correction methods using time delay estimation on RF signals critically depends on the spatial covariance measured across the array. The estimation accuracy decreases as the covariance decreases. The van Cittert-Zernike theorem applied to pulse echo ultrasound implies that the spatial covariance measured by correlation varies

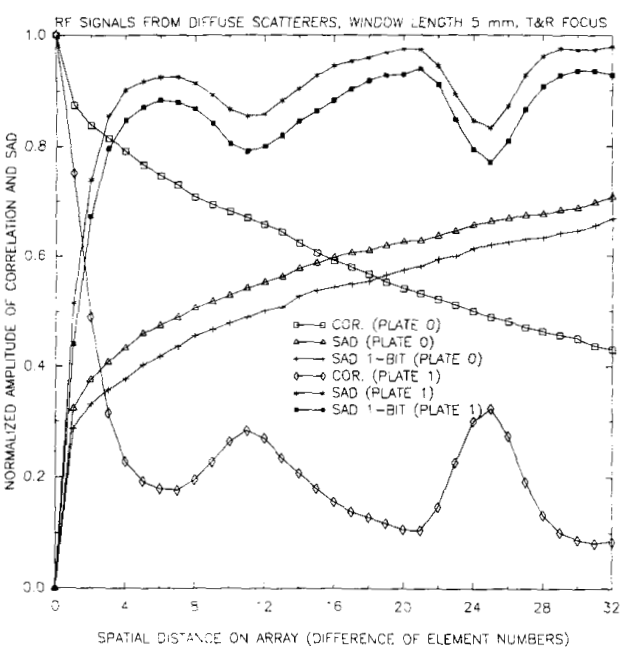

Fig. 6. Correlation and SAD functions across the array.

linearly across the array [18]. To compare the performance of correlation and SAD techniques, the correlation and SAD functions across the array are calculated using the spatially separated RF signals of Plate 0 and Plate 1 (see Fig. 6).

Calculations are performed on RF signals from different pairs of array elements. The spatial separation is expressed as the number of elements between the pair. In the computations, SAD and normalized correlation expressions given in (1) and (8), respectively, are employed on RF signals. These signals are reflected from diffuse scatterers over a single scan line within an axial window (size of $5 \mathrm{~mm}$ ) located at $60 \mathrm{~mm}$. The transmit focus is at $60 \mathrm{~mm}$ and dynamic receive focusing is used. Correlation and SAD functions are computed at zero time lag, i.e., at zero shift index. The results of different element pairs with equal spatial distance are averaged. The overall results for correlation and $\mathrm{SAD}$ are normalized by their maximum values. The maximum value of the correlation function is achieved at zero separation whereas the maximum for SAD is obtained at 63 elements difference. Therefore, SAD values for pairs are not directly comparable to their correlation counter parts in quantitative terms. However, the value of the SAD function for adjacent elements (1 element difference) forms a good basis for comparison purposes.

Fig. 6 depicts the results for zero to 32 element separation corresponding to one half of the aperture. The figure shows that the correlation function varies linearly across the array as the van Cittert-Zernike theorem implies [18]. It can be observed that incremental changes in both functions are quite similar for element differences more than one. The figure indicates that the dependence of both functions on phase aberration is also comparable. The figure also shows that the difference in full word SAD and 1-b SAD functions is minor.

Since aberration correction using $S A D$ and correlation is based on estimation of relative phase errors, degradation in performance due to inactive elements is expected to be similar [8]. It is observed from Fig. 6 that the SAD of RF signals spatially separated up to 6 elements is sufficiently low for phase estimation. Hence, SAD technique can be used for phase 
correction in the presence of inactive, neighboring elements up to about $10 \%$ of overall array elements. This correction can be achieved by estimating relative phase aberration between the nearest active elements, and interpolating relative aberration of inactive elements between them [19]. Thus degradation in estimation performance due to inactive elements can be reduced.

In SAD-based aberration correction, each relative phase estimation is independently performed, and the aberration pattern across the array is obtained by cumulative summation of these relative measurements as expressed in Eq. 3. This results in accumulation of errors in relative phase measurements across the array. Large errors can be imposed by the reduced covariance between RF signal pairs due to significant degradation in transmit beamforming performance. This is the main reason for iterations required in phase estimation, and for instability of the 1-b SAD estimator. The improved performance of averaged SAD estimators over others is simply the result of reduced estimation errors from averaging.

Truncation of wordlength to $1 \mathrm{~b}$ reduces the absolute difference operation to a bitwise EXCLUSIVE-OR logic operation. This significantly decreases computations required in SAD. Noting that 1-b multiplication is equivalent to a bitwise AND logic operation, the unnormalized correlation function can be represented by the sum of AND operations of 1-b RF samples whereas such a simple relation cannot be obtained for the 1-b normalized correlation because of the normalization process.

\section{RESUltS AND CONCLUSIONS}

A new phase aberration correction scheme based on SAD is presented for phased array ultrasound imaging. Performance of the proposed method has been tested with experimental data. The method can be used for estimation of the aberration pattern from echo signals reflected by line targets and/or diffuse scatterers. Computational complexity of the SAD method is relatively low since it involves only additions, and the overhead due to averaging is insignificant. The computational efficiency is further improved using a single bit wordlength. Dead elements in the array can decrease the accuracy of phase estimation using SAD minimization, and the degradation in the estimation performance is expected to be similar to that for cross correlation [8].

The SAD method involves sampling of RF echo signals received from all array channels, where the accuracy of the estimation is limited by the sampling rate. This is a major drawback in all digital, real-time phase correction schemes as well as the one discussed in this paper. The sampling rate can be increased using an interpolation technique, prior to phase estimation. Since timing accuracy in phase estimation is more critical than amplitude accuracy, a simple interpolation scheme such as bandpass linear interpolation can be a cost effective, real-time solution.

Finally, we conclude that phase aberration correction using SAD minimization with a single bit wordlength and averaging estimated phase patterns over a number of scan angles compares favorably to computationally more complex methods, whereas it lends itself to real-time implementation through a simple architecture.

\section{REFERENCES}

[1] G. E. Trahey, P. D. Freiburger, L. F. Nock, and D. C. Sullivan, "In vivo measurements of ultrasonic beam distortion in the breast," Ultrason. Imaging, vol. 13, pp. 71-90, 1991.

[2] M. Hirama and T. Sato, "Imaging through an inhomogeneous layer by least-mean-square error fitting," $J$. Acoust. Soc. Amer., vol. 75, pp. 1142-1147, Apr. 1984.

[3] S. W. Smith and G. E. Trahey and O. T. von Ramm, "Phased array ultrasound imaging through planar tissue layers," Ultrasound Med. Biol., vol. 12, pp. 229-243, Mar. 1986.

[4] S. W. Flax and M. O'Donnell, "Phase-aberration correction using signals from point reflectors and diffuse scatterers: basic principles," IEEE Trans. Ultrason. Ferroelec. Freq. Contr., vol. 35, pp. 758-767, Nov. 1988.

[5] D. Rachlin, "Direct estimation of aberrating delays in pulse-echo imaging systems," J. Acoust. Soc. Amer., vol. 88, pp. 191-198, July 1990.

[6] D. Zhao and G. E. Trahey, "Comparisons of image quality factors for phase aberration correction with diffuse and point targets," IEEE Trans. Ultrason. Ferroelec. Freq. Contr., vol. 38, pp. 125-132, Mar. 1991.

[7] F. Wu, M. Fink, R. Mallart, J. L. Thomas, N. Chakroun, D. Cassereau, and C. Prada, "Optimal focusing through aberrating media: a comparison between time reversal mirror and time delay correction techniques," in Proc. 1991 IEEE Ultrason. Symp., 1991, pp. 1195-1199.

[8] G. E. Trahey and P. D. Freiburger, "An evaluation of transducer design and algorithm performance for two dimensional phase aberration correction," in Proc. 1991 IEEE Ultrason. Symp., 1991, pp. 1181-1187.

[9] M. O'Donnell and P. C. Li, "Aberration correction on a two-dimensional anisotropic phased array," in Proc. 1991 IEEE Ultrason. Symp., 1991, pp. 1189-1193.

[10] R. Kanda, Y. Sumino, K. Takamizawa, and H. Sasaki, "An investigation of wavefront distortion correction: Correction using averaged phase information and the effect of correction one and two dimensions," in Proc. 1991 IEEE Ultrason. Symp., 1991, pp. 1201-1206.

[11] K. Scarbrough, N. Ahmed and G. C. Carter, "On the simulation of a class of time delay estimation algorithms," IEEE Trans. Acoust. Speech, Signal Process., vol. ASSP-29, pp. 534-539, June 1981.

[12] ,D. I. Barnea and H. F. Silverman, "A class of algorithms for fast digital image registration," IEEE Trans. Comput., vol. C-21, pp. 179-186, Feb. 1972.

[13] P. Maragos, "Morphological correlation and mean absolute error criteria," in Proc. 1989 Int. Conf. on Acoust., Speech, and Signal Process., 1989 , pp. $1568-1571$

[14] L. N. Bohs and G. E. Trahey, "A novel method for angle independent ultrasonic imaging of blood flow and tissue motion," IEEE Trans. Biomed. Eng., vol. 38, pp. 280-286, Mar. 1991.

[15] B. D. Steinberg and E. Yadin, "Effect of multipath and scattering on array gain of large adaptive beam forming array,"IEEE Trans. Antennas Propagat., vol. AP-33, pp. 481-487, May 1985.

[16] M. Karaman, A. Atalar, and H. Köymen, "Adaptive digital beamforming for real-time phased array ultrasound imaging," in Proc. 1991 IEEE Ultrason. Symp., 1991, pp. 1207-1210.

[17] M. O'Donnell and S. W Flax, "Phase-aberration correction using signals from point reflectors and diffuse scatterers: experimental results," IEEE Trans. Ultrason. Ferroelec, Freq. Contr, vol. 35, pp. 768-774, Nov. 1988

[18] R. Mallart and M. Fink, "The van Cittert-Zernike theorem in pulse-echo measurements." J. Acoust. Soc. Amer., vol. 90, pp. 2718-2727, 1991.

[19] M. O'Donnell and W. E. Engeler, "Correlation-based aberration correction in the presence of inoperable elements," IEEE Trans. Ultrason. Ferroelec. Freq. Contr., vol. 39, pp. 700-707, Nov. 1992.

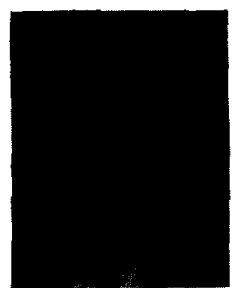

Mustafa Karaman (S'88) was born in Ballkesir, Turkey, in 1964. He received the B.Sc. degree from Middle East Technical University, Ankara Turkey, and the M.Sc. degree from Bilkent University, Ankara, Turkey, in 1986 and 1988, respectively, both in electrical and electronics engineering. $\mathrm{He}$ is currently studying toward the Ph. D. degree in electrical and electronics engineering at Bilkent University.

He has been a Research Assistant at Bilkent University since 1986. His current research interests include VLSI signal processing and medical ultrasound imaging. 


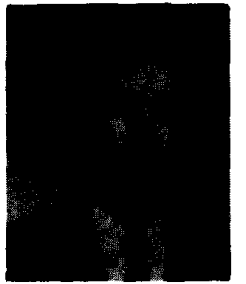

Abdullah Atalar (M'88-SM'90) was bom in Gaziantep, Turkey, in 1954 . He received the B. $\mathrm{S}$. degree from Middle East Technical University, Ankara, Turkey in 1974, and the M. S. and Ph. D. degrees from Stanford University, Stanford, CA in 1976 and 1978, respectively, all in Electrical Engineering.

From 1978 to 1980 he was first a Post Doctoral Fellow and later an Engineering Research Associate in Stanford University working on acoustic microscopy. For eight months he was with Hewlett Packard Labs, Palo Alto, CA, engaged in photoacoustics research. From 1980 to 1986 he was on the faculty of the Middle East Technical University as an Assistant Professor. From 1982 to 1983 on leave from University, he was with Ernst Leitz Wetzlar, Wetzlar, West Germany, where he was involved in the development of the commercial acoustic microscope In 1986 he joined the Bilkent University as the Chairman of the Electrical and Electronics Engineering Department and served in the founding of the Department where he is currently a Professor. His current research interests include acoustic imaging, linear acoustics, and computer-aided design in Electrical Engineering.

Dr. Atalar he was awarded the $1984 \mathrm{H}$. Tuugaç Foundation Award of TUB, ITAK, Turkey for his contributions to acoustic microscopy.

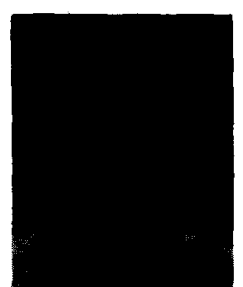

Hayrettin Köymen (S'74-M'87-SM'91) was born in Ankara, Turkey, on June 7, 1952. He received the B.Sc. and M.Sc degrees from Middle East Technical University, Ankara, Turkey, in 1973 and 1976, respectively, and the $\mathrm{Ph}$. D. degree from the University of Birmingham, Birmingham, England in 1979 , all in electrical engineering.

He became a faculty member of the Middle East Technical University in 1979. Until 1982 his work involved underwater acoustics and oceanographic instrumentation. Since 1982, his work has involved physiological signal acquisition and processing, medical ultrasonics and ultrasonic nondestructive evaluation. In 1990, he joined the faculty of Bilkent University, Ankara, where he is now a Professor in the Department of Electrical and Electronics Engineering. His current research interests are personal computer-based biomedical signal acquisition and processing, medical imaging, finite amplitude effects in medical ultrasonics, and acoustic microscopy using leaky waves in layered media.

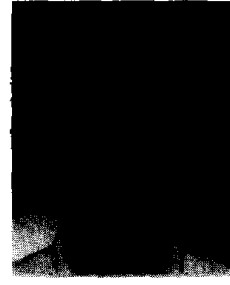

Matthew O'Donnell (M'79-SM'84-F'93) received the B.S. and Ph.D. degrees in physics from the University of Notre Dame in 1972 and 1976, re spectively.

He was a Postdoctoral Fellow at the Physics Department, Washington University, working on applications of ultrasonics to medicine and nondestructive testing. He was subsequently a Senior Research Associate in the Physics Department and Research Instructor of Medicine with the Department of Medicine. In 1980, he joined Research and Development, General Electric Corporation, Schenectady, NY, where he worked on medical electronics, including NMR and ultrasound imaging systems. During 1984-1985, he was a Visiting Fellow with the Department of Electrical Engineering, Yale University, where he investigated automated image analysis systems. More recently, he has worked on the application of advanced VLSI circuits to medical imaging systems. In 1990, he became a Professor of Electrical Engineering and Computer Science with the University of Michigan at Ann Arbor. He has authors sixty-six publications and currently holds 31 patents.

Dr. O'Donnell is a member of Sigma Xi and the American Physical Society. He has received two Best Paper Awards. 